\title{
Diagnosis of apical periodontitis in root-filled teeth
}

\author{
Peter Jonasson $^{1} \cdot$ Thomas Kvist $^{1}$ iD
}

Received: 25 March 2018 / Accepted: 23 April 2018 / Published online: 15 May 2018

(C) The Author(s) 2018

\begin{abstract}
Root filled teeth are common in adult populations worldwide. When a root-filled tooth causes swelling and/or pain it is usually a sign of infection. In combination with the presence of a periapical bone lesion visible in an intraoral radiograph the diagnosis of apical periodontitis is usually quite straightforward. However, when pain is present but radiographic signs are absent or, in particular, when the tooth is asymptomatic but signs of disease are present at an X-ray the diagnostic process is associated with several uncertainties. Asymptomatic periapical bone lesions indicating apical periodontitis is common in root-filled teeth. Based on the current state of knowledge, it is reasonable to assume that the condition does not pose a serious health problem at a population level. Therefore, it is reasonable for the clinician, in most situations, to adopt a diagnostic strategy that helps to refrain from overdiagnosis. Otherwise many patients will risk costly retreatments or even tooth extractions without significant benefit.
\end{abstract}

Keywords Apical periodontitis - Root-filled tooth · Infectious disease · Radiographic lesion · Differential diagnosis

\section{Quick reference/description}

Root-filled teeth are common in adult populations worldwide. When a root-filled tooth causes swelling and/or pain, it is usually a sign of infection. In combination with the presence of a periapical bone lesion visible in an intraoral radiograph, the diagnosis of apical periodontitis is usually quite straightforward. However, when

Thomas Kvist

kvist@odontologi.gu.se

1 Department of Endodontology, Institute of Odontology, Sahlgrenska Academy, University

of Gothenburg, Gothenburg, Sweden 
pain is present, but radiographic signs are absent or, in particular, when the tooth is asymptomatic, but signs of disease are present at an X-ray, the diagnostic process is associated with several uncertainties. Asymptomatic periapical bone lesions indicating apical periodontitis are common in root-filled teeth. Based on the current state of knowledge, it is reasonable to assume that the condition does not pose a serious health problem at a population level. Therefore, it is reasonable for the clinician, in most situations, to adopt a diagnostic strategy that helps to refrain from overdiagnosis. Otherwise, many patients will risk costly retreatments or even tooth extractions without significant benefit.

\section{Symptoms}

- Soreness

- Dull pain

- Discomfort for chewing

- Swelling

- Systemic symptoms (fever, malaise, and lymphadenopathy).

\section{Clinical examination}

A medical and dental history of the patient is a prerequisite for clinical examination. For the root-filled tooth in question, we observe for the following:

- Any signs of inflammation in surrounding bony structures

- Swelling

- Redness

- Tenderness

- Sinus tracts

- Periodontal pocket

- Caries

- Defective restorations.

\section{Materials/instruments}

Standard equipment for clinical dental examination (probe, mirror....) Loupes or microscope.

\section{Radiographic examination}

- Intraoral radiographs from different angulations.

- Cone-beam computed tomography in certain situations. 


\section{Procedure}

Common scenarios and diagnostic strategy to avoid overdiagnosis of apical periodontitis (AP).

\begin{tabular}{lll}
\hline & $\begin{array}{l}\text { Clinical signs of AP in } \\
\text { a root-filled tooth }\end{array}$ & $\begin{array}{l}\text { Intraoral radiographic signs } \\
\text { of AP in a root-filled tooth }\end{array}$ \\
\hline Scenario 0 & Not present & Not present \\
Scenario I & Present & Present \\
Scenario II & Present & Not present \\
Scenario III & Not present & Present \\
\hline
\end{tabular}

\section{Scenario 0. An asymptomatic root-filled tooth without signs of AP on intraoral radiographs}

AP is absent. No indication for further examination.

\section{Scenario I: A symptomatic root-filled tooth with signs of AP on intraoral radiographs}

$\mathrm{AP}$ is present.

\section{Scenario II: A symptomatic root-filled tooth without signs of AP on intraoral} radiographs

Step 1. Consider first other odontogenic origins of pain.

- Neighboring teeth

- Vertical root fracture

- Pulpitis in untreated parts of the root canal system

- Periodontal abscess

- Apical fenestration.

Step 2. If no signs of other odontogenic origin are found consider nonodontogenic origin of pain.

- Referred pain from temporomandibular disorder

- Maxillary sinusitis (if from a distal tooth in upper jaw)

- Atypical odontalgia ("Phantom Tooth Pain")

- Trigeminal neuralgia or other neuropathic pain condition.

Step 3. If no other diagnosis may explain the pain consider a cone-beam computed tomography (CBCT) scan. 
- If an apical radiolucency is observed in the scan, then AP is diagnosed as present.

- If no bone destruction is seen in the CBCT scan, reconsider other diagnoses (Step 1 and 2) that may mimic the symptoms of AP.

\section{Scenario III: An asymptomatic root-filled tooth with signs of AP on intraoral radiographs}

Step 1. Determine the time, since root filling was placed.

Step 2. Assess the size of the radiolucency in, if available, compare with the previous radiographic examinations.

Step 3. Judge the quality of the root filling.

If an intraoral radiographic examination reveals a clearly visible apical radiolucency that has remained unchanged, increased in size, or emerged after a period of 4 years after root canal treatment, this strongly indicates persisting infection and that AP is present. If the quality of root filling is also poor, the diagnosis is almost infallible.

\section{Situations of particular ambiguity}

A. Time since root filling $\leq 4$ years and a radiolucency is still present. May still heal without further invention in particular if root-filling quality is good. No diagnosis of AP. Suggest re-examination within 1-2 years.

B. Time since root filling $>4$ years and a widening of the periodontal ligament is present. Great uncertainty about the nature of the lesion, in particular, if rootfilling quality is good. No diagnosis of AP. No further examinations indicated.

C. Time since root filling $>4$ years and clinician is uncertain about a possible lesion. Consultation with a colleague or radiologist is recommended.

D. Time since root filling $>4$ years and the radiolucency is still present but reducing in size continuously. Further healing potential may be present especially if rootfilling quality is good.

\section{Differential diagnosis}

\section{Clinical}

- Vertical root fracture

- Pulpitis in a root-filled tooth due to missed root canal

- Periodontal abscess

- Apical fenestration

- Maxillary sinusitis (if from a distal tooth in upper jaw)

- Atypical odontalgia ("Phantom Tooth Pain") 
- Referred pain from temporomandibular disorder

- Trigeminal neuralgia and other neuropathic pain conditions.

\section{Radiographic}

- Fibroosseous lesions

- Ameloblastomas

- Nasopalatine duct cysts

- Lateral periodontal cyst

- Keratocystic or other odontogenic tumor

- Primary or metastatic malignancies

- Systemic disease manifestations, for example osteoporosis.

\section{Pitfalls and complications}

- Asymptomatic small lesions are very common among root-filled teeth.

- It is difficult to determine the exact time required for the healing of apical periodontitis in a root-filled tooth.

- It is difficult to determine what should be considered as a sufficient healing of bone destruction to constitute successful outcome of root canal treatment.

- Very little is known about potential risks of local or systemic adverse effects due to untreated apical periodontitis in root-filled teeth.

\section{Further reading}

1. T. Kvist (ed.) Diagnosis. In: Apical periodontitis in root-filled teeth. https://doi. org/10.1007/978-3-319-57250-5_5.

2. Pasqualini D, Scotti N, Ambrogio P, Alovisi M, Berutti E. Atypical facial pain related to apical fenestration and overfilling. Int Endod J. 2012; 45(7):670.

3. Rosen E, Taschieri S, Del Fabbro M, Beitlitum I, Tsesis I. The diagnostic efficacy of cone-beam computed tomography in endodontics: a systematic review and analysis by a hierarchical model of efficacy. J Endod. 2015;41:1008-14.

4. Kruse C, Spin-Neto R, Wenzel A, Kirkevang LL. Cone-beam computed tomography and periapical lesions: a systematic review analyzing studies on diagnostic efficacy by a hierarchical model. Int Endod J. 2015;48:815-28.

5. Pak JG, Fayazi S, White SN. Prevalence of periapical radiolucency and root canal treatment: a systematic review of cross-sectional studies. J Endod. 2012;38:1170-6.

Open Access This article is distributed under the terms of the Creative Commons Attribution 4.0 International License (http://creativecommons.org/licenses/by/4.0/), which permits unrestricted use, distribution, and reproduction in any medium, provided you give appropriate credit to the original author(s) and the source, provide a link to the Creative Commons license, and indicate if changes were made. 